\title{
Microfocused Ultrasound for Nonablative Skin and Subdermal Tightening to the Periorbitum and Body Sites: Preliminary Report on Eighty-Two Patients*
}

\author{
Gordon H. Sasaki ${ }^{1 \#}$, Ana Tevez ${ }^{2}$ \\ ${ }^{1}$ Loma Linda University Medical School Private Practice, Pasadena, USA; ${ }^{2}$ Sasaki Advanced Aesthetic Medical Center, Pasadena, \\ USA. \\ Email: " ghsasakimd@drsasaki.com
}

Received June $30^{\text {th }}, 2012$; revised July $31^{\text {st }}, 2012$; accepted August $10^{\text {th }}, 2012$

\begin{abstract}
Lax crepey skin is a major unsightly finding throughout the face and body that occurs from chronological aging and environmental photodamage. Surgical lifting procedures are unable to induce sufficient neocollagenesis and also associated with visible scars, risks and recovery time. In this preliminary report, non-invasive delivery of microfocused ultrasonic thermal coagulation points at two levels of the dermis outside the orbital rims was effective in reduction of crepey, wrinkled and sagging skin for about $1 \frac{1}{2}$ years in nineteen treated patients. Matched Orientation Mirror Imaging produced an average brow height elevation of $1-2 \mathrm{~mm}$. Microfocused thermal coagulation points at multiple tissue levels also induced tissue tightening and reduction of crepey wrinkled skin in the décolletage, brachium, periumbilicus, inner thigh and knees. Investigator and Subject Global Aesthetic Improvement Scale graded responses that correlated to the severity of the degree of crepiness and laxity. Pain management during the procedure included the use of infiltration of local anesthesia. No permanent adverse events were observed. Further innovations in the current technology are needed for more effective and safer delivery of energy to improve the appearance of crepey and lax tissue in the head, neck and body.
\end{abstract}

Keywords: Crepey; Focused; Imaged; Ultrasound; Noninvasive; Lifting; Tightening

\section{Introduction}

Microfocused ultrasound energy has been shown to lift and tighten facial and neck skin and subdermal tissue $[1,2]$ by delivering focal thermal coagulation points (TCP) below the skin's surface and targeting foundational layers without affecting intervening tissue [3-6]. These special characteristics present a suitable alternative treatment for thin, crepey and wrinkled eyelid skin outside the orbital rims, inner arm, décolletage, periumbilicus, inner thighs and knees whose management remains resistant to current modalities [7-10]. With the recent development of a $1.5 \mathrm{~mm}$ focal depth transducer, which deposits energy more superficially compared to the deeper $3.0 \mathrm{~mm}$ and $4.5 \mathrm{~mm}$ focal depth transducers, precise heating at three levels is possible now and may provide optimal temperatures for increased collagenesis and elastogenenesis to renew crepey skin.

The purposes of this contribution were 1) To evaluate

\footnotetext{
${ }^{*}$ Ulthera provided an unrestricted educational grant which was used to support publication costs.

${ }^{\#}$ Corresponding author.
}

the effects of dual levels of treatment depths to the dermis surrounding the eyelid skin outside the orbital rims; and 2) To report on the safety and efficacy to body sites with dermal and subdermal treatment depths.

\section{Ultrasound System}

The focused ultrasound device (FDA-approved September 2009) incorporates on a user interface screen in the visualization mode an ultrasound image of tissue layers to $8 \mathrm{~mm}$ depth. On activation of the selected transducer on the skin surface, a horizontal treatment line is displayed either at a $1.5 \mathrm{~mm}$ depth within the upper reticular dermis, $3.0 \mathrm{~mm}$ depth in the lower reticular dermis, or $4.5 \mathrm{~mm}$ depth within the subdermal levels, as determined by the present fixed focal depth of the $1.5 \mathrm{~mm}, 3.0 \mathrm{~mm}$ or $4.5 \mathrm{~mm}$ transducers. One of three transducers was chosen to achieve the designated treatment depths and desired amounts of distributed ultrasound energy (joule)/TCP: 1) DS $10-1.5 \mathrm{~mm}, 19 \mathrm{MHz}(0.15-0.25$ joules/TCP); 2) DS 7 - $3.0 \mathrm{~mm}, 7 \mathrm{MHz}(0.25-0.45$ joules/TCP; 3$)$ DS 4 - $4.5 \mathrm{~mm}, 4 \mathrm{MHz}(0.75-1.2$ joules/TCP. On activation 
through contact gel, the $1.5 \mathrm{~mm}$ and $3.0 \mathrm{~mm}$ transducers released a maximum of $23 \mathrm{TCPs}$, spaced about $1.1 \mathrm{~mm}$ apart along a $25 \mathrm{~mm}$ line in the superficial and deeper level of the dermis, respectively. Likewise, the $4.5 \mathrm{~mm}$ transducer delivered a maximum of $17 \mathrm{TCPs}$, spaced 1.5 $\mathrm{mm}$ apart, along a $25 \mathrm{~mm}$ line in the deeper subdermal plane. About ninety-five percent of the vibratory energy creates friction between molecules, which produces an optimal $60^{\circ} \mathrm{C}-70^{\circ} \mathrm{C}$ in a confined approximate $0.5 \times 1.8$ $\mathrm{mm}$ zone (TCP), depending on the transducer used, for collagen denaturation and eventual tissue tightening [11].

For treatment of crepey skin outside the orbital rims, the operator used a dedicated DS $10-1.5 \mathrm{~mm}, 10 \mathrm{MHz}$ transducer $(0.15-0.25$ joules/TCP) and a DS $7-3.0 \mathrm{~N}$ $\mathrm{mm}$ narrow transducer $(0.25-0.45$ joules/TCP) which delivered a shorter treatment line of $14 \mathrm{~mm}$ (13 TCPs) to accommodate the anatomical boundaries of this treatment site. In all other areas, the operator dispensed the maximum treatment length of $25 \mathrm{~mm}$ and the highest amount of joules/TCP for potential maximal results.

\section{Patient Selection}

Adult patients were recruited from the site's data base. Inclusion criteria included mild-to-moderate crepey skin outside the orbital rims. These patients also exhibited mild-to-moderate ptosis of skin folds and orbital portions of the underlying fibro-muscular layer orbicularis oculi muscle. Patients with crepey skin at the décolletage, inner arm, periumbilicus, buttocks inner upper thighs, knees and hands were also included for treatment. Exclusion criteria included active local infections or skin diseases that might alter wound healing; acne or keloidal scarring; significant ptotic skin or subcutaneous fat; pregnant or breast-feeding women; recent ablative or nonablative skin procedures; and surgical procedures within a year to the proposed treatment sites.

\section{Photographic and Statistical Analysis}

A custom-designed Canfield photographic and lighting system (Canfield Scientific, Inc., Fairfield, NJ) was used for baseline and follow-up standardized photography. Matched Orientation Function of Mirror Software compared baseline to post-treatment distances $(\mathrm{mm})$ between reference points for brow on a standardized facial positioning table for the vertical displacement of each brow (midpupil, lateral canthus, lateral tail of brow) from the intercanthal horizontal axis for the upper periorbital treatments. Each photographic image was automatically tagged with a specific label (metadata) that could not be altered. In each patient, the average of three vertical displacements of each brow (midpupil, lateral canthus, lateral tail of brow) from the intercanthal horizontal axis provided the database for comparison measurements for each subject. Disadvantages of employing the brow as a reference point for measurements were the inherent vagaries for mobile groomed structures. Standardized Photography compared baseline and post-treatment changes in crepiness to the upper and lower periorbital skin and the aforementioned body sites. The validated Fitzpatrick Wrinkle, Fold and Tissue Laxity Scale [12] was used to classify and score patients by two independent investigators: Class I mild, 1 - 3 score; Class II moderate, score 4 - 6; class III severe, score 7 - 9. Aesthetic efficacy from baseline to six months was rated by the same two independent investigators using the Investigator Global Aesthetic Improvement Scale (IGAIS) from standardized photographs $(0=$ no change; 1 = mild improvement; 2 = moderate improvement; and 3 = significant improvement. Patients used a Subject Global Aesthetic Improvement Scale (SGAIS): $(0=$ no change; $1=$ mild changes; $2=$ moderate changes; $3=$ significant changes) to assess their results at the six month evaluation period. During their treatments, patients assessed their levels of heat-pain perception on a 10 -point scale $(0=$ no pain; $1-4=$ mild pain; $5-8=$ moderate pain; 9 - $10=$ severe pain).

\section{Treatment Protocol}

The usage of topical skin care products such as isotretinoin, glycolic and salicylic acids was discontinued about two weeks prior to treatment. Patients were reminded not to apply facial creams, lotions, powders and foundations on the treatment day. Patients washed their faces with a mild cleanser just prior to their procedure. All metal jewelry was removed from the facial area. Patients with a history of viral infections were placed on prophylactic antivirals two days before and six days after the procedure. Treatment was not recommended directly over those areas with mechanical implants or electrical devices and with soft tissue augmentation material.

After a thin layer of ultrasound transmission gel was applied to the transducer's window, the selected transducer was positioned on the designated treatment sector and activated for imaging of the skin and subdermal structures. On activation of the transducer, a series of thermal coagulation points were deposited at the selected tissue level in a straight line. This sequence was repeated within each treatment sector with the selected number, direction and depth of treatment lines, as recommended in the individualized maps (see below). The refrigerated ultrasound transmission gel was frequently reapplied to the transducer's window to ensure proper tissue imaging and coupling.

For patients who acknowledged their low pain threshold levels or experienced moderate discomfort during treatment, a pain management program was initiated in a 
graded fashion beginning with oral non-steroidal anti-inflammatory drugs, pain and sedative medications, distractive hand/foot massages, reducing skin temperatures by an air coolant device, lowering the energy settings either by one level for each transducer, or shortening the length of treatment lines. The usage of topical analgesic gels for an hour prior to treatment lessened pain in a few patients. Finally, infiltration of buffered lidocaine was offered for pain relief during treatment in sensitive sites such as the décolletage, inner arms, hands, abdomen, upper inner thighs or knees.

\section{Peri-Orbital Treatment Map}

The treatment map recommended for the periorbital area consists of delivering 15 radial lines ( $14 \mathrm{~mm} /$ line) across the upper lid-brow complex, 15 cris-crossing lines at the crow's feet area, and 15 radial lines below the lower lid margin with the DS $7-3.0 \mathrm{Nmm}$ transducer (13 TCPs/line, 0.45 joules/TCP), distributing a total of 263.2 joules within the lower level of the dermis (Figure 1). Thereafter, the upper dermis was treated with the DS 10 $1.5 \mathrm{Nmm}$ transducer (13 TCPs/line, 0.25 joules/TCP) that delivered 15 radial lines $(14 \mathrm{~mm} /$ line $)$ across the upper lid-brow complex, 15 cris-crossing lines at the crow's feet area, and 15 radial lines below the lower lid margin, distributing a total of 146.25 joules. Treatment of periorbital skin at a dual depth was limited to tissue only over the orbital portion of the orbicularis muscle and bone in order to prevent intraocular injury. Currently, there are no commercially available eye shields that have been shown to effectively block ultrasound energy.

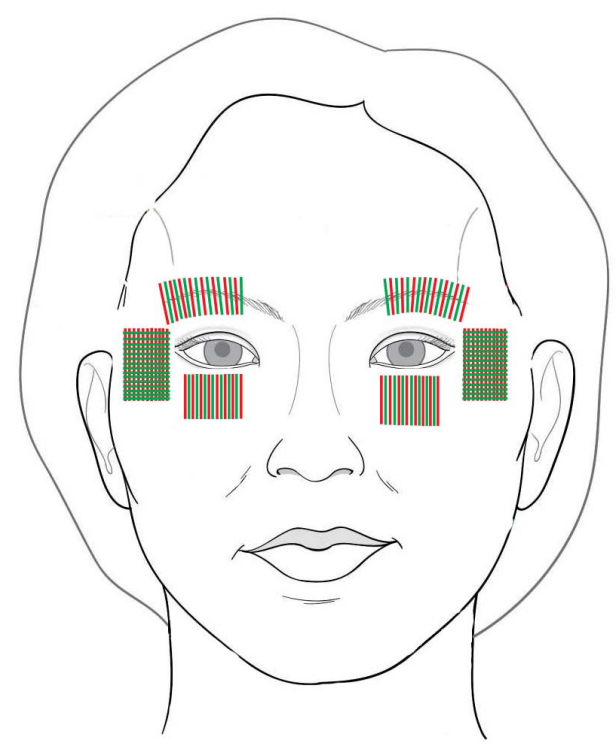

Figure 1. Periorbital Treatment Map with the DS 7 - 3.0 Nmm (13 TCPs/14 mm line) and DS 10 - 1.5 Nmm (13 TCPs/14 mm line) transducers.

\section{Body Treatment Map}

The treatment maps recommended for the body varied by area sizes, depths of dual treatment, and deposited total energy, as shown in Figure 2. Dual treatment began at the subdermal depth and then proceeded to the more superficial dermal level with all treatment lines placed in a vertical (foot-to-head) direction, as listed below in Table 1.

\section{Results}

A total of eighty-two patients ( 81 females) were treated and evaluated, as listed in Table 2. The median age was 51.8 years (range $30-72$ years). Ethnic backgrounds included 42 Caucasians, 31 Hispanics, 8 Asians, and 1 African-American. Treatment sites comprised the periorbitum, décolletage, brachia, abdomen, inner thighs, knees, hands and buttocks.

Primary outcomes for efficacy of eyebrow lifting after treatments within the upper and lower levels of the dermis were measured by Matched Orientation Function of Mirror Software, comparing baseline to 6 month posttreatment changes $(\mathrm{mm})$ between reference points. A single treatment produced an average elevation between $1-2 \mathrm{~mm}(7 \%-8 \%$ increase from baseline) in each 19 patients, as depicted in Figure 3 and Figure 4.

Two masked evaluators classified and scored patients' skin and subdermis at the eight sites based on the following clinical gradations of crepiness and ptotic folds: Class I mild, score 1 - 3; Class II moderate 4 - 6. Patients, who presented with Class III severe scores 7 - 9, were excluded from treatments. Investigators and patients compared changes from baseline to six month photographs, listed in Table 3

In general, wrinkled and crepey skin of lower grading (Class I and II) in the periorbital region, inner brachium, periumbilicus and knees experienced moderate improvements by IGAIS and SGAIS assessment at 6 months (Figures 5-8). Salutary effects were observed as early as 6 weeks (especially to treated eyelid and periorbital skin), but the majority of patients appreciated a smoothing and tightening of the crepey skin between 3 to 6 months. Observed responses lasted about from 6 months to $1 \frac{1}{2}$ years after a single treatment. In the décolletage, inner thighs, hands and buttocks areas, the frequency of clinical responses was not as great, when compared to the numbers observed in the periorbital region, inner brachium, periumbilical, and knee areas.

\section{Side Effects and Complications}

All patients developed fleeting erythema immediately after treatment, especially around the upper and lower eyelids. All erythema dissipated within a few hours. Transient edema from therapy was difficult to assess because of 


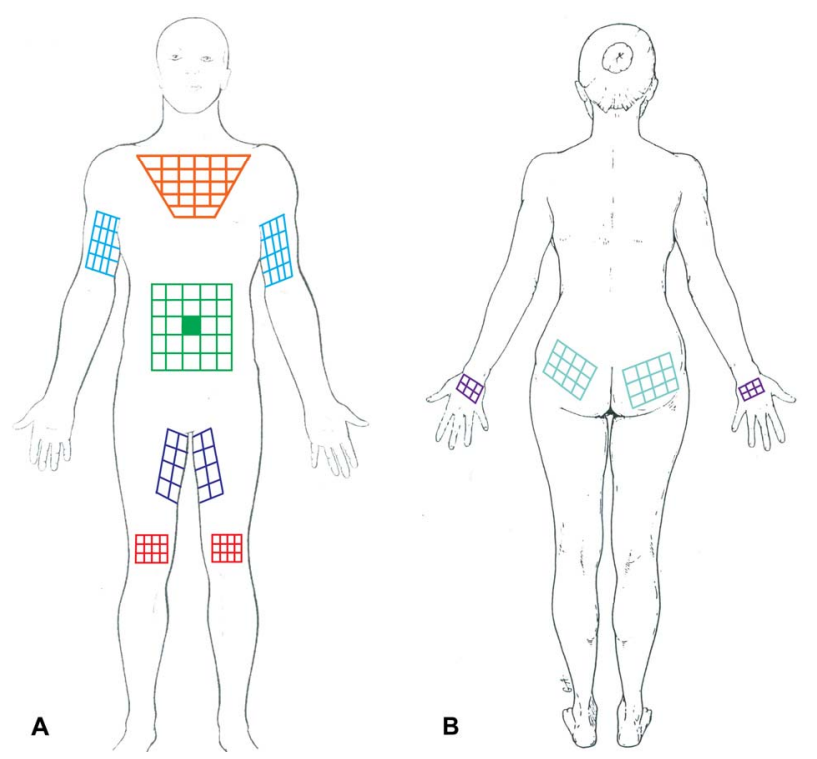

Figure 2. Body Treatment Maps with the DS 10 - $1.5 \mathrm{~mm}$ (23 TCPs/25 mm line), DS 7 - $3.0 \mathrm{~mm}$ (23 TCPs/25 mm line) and DS 4 $4.5 \mathrm{~mm}(17 \mathrm{TCP} / 25 \mathrm{~mm}$ line) transducers.

Table 1. Treatment maps for body sites.

\begin{tabular}{|c|c|c|c|c|}
\hline Site(s) & $\begin{array}{c}\text { Number of } \\
2.5 \times 2.5 \mathrm{~cm} \text { square }\end{array}$ & Transducers & $\begin{array}{l}\text { Number of Lines } \\
\text { (mm length)/square }\end{array}$ & $\begin{array}{c}\text { Total } \\
\text { Joules/Squares } \\
\end{array}$ \\
\hline Décolletage & 21 & $\begin{array}{l}\text { DS } 7-3.0 \mathrm{~mm} \\
\text { DS } 10-1.5 \mathrm{~mm}\end{array}$ & $\begin{array}{l}30(25 \mathrm{~mm}) \\
30(25 \mathrm{~mm})\end{array}$ & $\begin{array}{l}310.5 \\
172.5\end{array}$ \\
\hline Brachium & 16 & $\begin{array}{l}\text { DS } 4-4.5 \mathrm{~mm} \\
\text { DS } 7-3.0 \mathrm{~mm}\end{array}$ & $\begin{array}{l}30(25 \mathrm{~mm}) \\
30(25 \mathrm{~mm})\end{array}$ & $\begin{array}{c}612 \\
310.5\end{array}$ \\
\hline Periumbilicus & 24 & $\begin{array}{c}\text { DS } 4-4.5 \mathrm{~mm} \\
\text { DS } 7-3.0 \mathrm{~mm} \\
\text { DS } 10-1.5 \mathrm{~mm}\end{array}$ & $\begin{array}{l}30(25 \mathrm{~mm}) \\
30(25 \mathrm{~mm}) \\
30(25 \mathrm{~mm})\end{array}$ & $\begin{array}{c}612 \\
310.5 \\
172.5\end{array}$ \\
\hline Inner Thigh & 8 & $\begin{array}{l}\text { DS } 4-4.5 \mathrm{~mm} \\
\text { DS } 7-3.0 \mathrm{~mm}\end{array}$ & $\begin{array}{l}30(25 \mathrm{~mm}) \\
30(25 \mathrm{~mm})\end{array}$ & $\begin{array}{c}612 \\
310.5\end{array}$ \\
\hline Knee & 12 & $\begin{array}{l}\text { DS } 4-4.5 \mathrm{~mm} \\
\text { DS } 7-3.0 \mathrm{~mm}\end{array}$ & $\begin{array}{l}30(25 \mathrm{~mm}) \\
30(25 \mathrm{~mm})\end{array}$ & $\begin{array}{c}612 \\
310.5\end{array}$ \\
\hline Hand & 6 & DS $7-3.0 \mathrm{~mm}$ & $15(25 \mathrm{~mm})$ & 155.25 \\
\hline Buttock & 12 & $\begin{array}{l}\text { DS } 4-4.5 \mathrm{~mm} \\
\text { DS } 7-3.0 \mathrm{~mm}\end{array}$ & $\begin{array}{l}30(25 \mathrm{~mm}) \\
30(25 \mathrm{~mm})\end{array}$ & $\begin{array}{c}612 \\
310.5\end{array}$ \\
\hline
\end{tabular}

subcutaneous injections of local anesthetic solution for pain management. No other adverse events (such as blistering, ulceration, scarring, dyschromia, numbness, bruising, or striations) were noted at any time point.

In most cases, patients completed treatments to skin outside the orbital rims by implementing the previously mentioned steps for pain-management. Unresponsive patients to these conservative antipain measures may benefit from selective nerve blocks to the supratrochlear, supraorbital, zygomaticotemporal, zygomatico-facial or infraorbital nerves for pain relief. Selective nerve blocks are preferable to local infiltration techniques which potentiates periorbital swelling and edema. In contrast, patients requested infiltration of local anesthesia to body
Table 2. Demographics of treatment groups.

\begin{tabular}{ll}
\hline Number (Sex) & 81 Females 1 Male \\
\hline Age & 51.8 years (range $30-72)$ \\
& 42 Caucasians \\
& 31 Hispanics \\
Ethnicity & 8 Asians \\
& 1 African American \\
& 19 (Periorbitum) \\
& 5 (Décolletage) \\
& 44 (Brachium) \\
& 1 (Hand) \\
& 6 (Periumbilicus) \\
Number of Patients & 2 (Buttock) \\
(Treatment Area) & 1 (Inner Thigh) \\
& 4 (Knee) \\
\hline
\end{tabular}



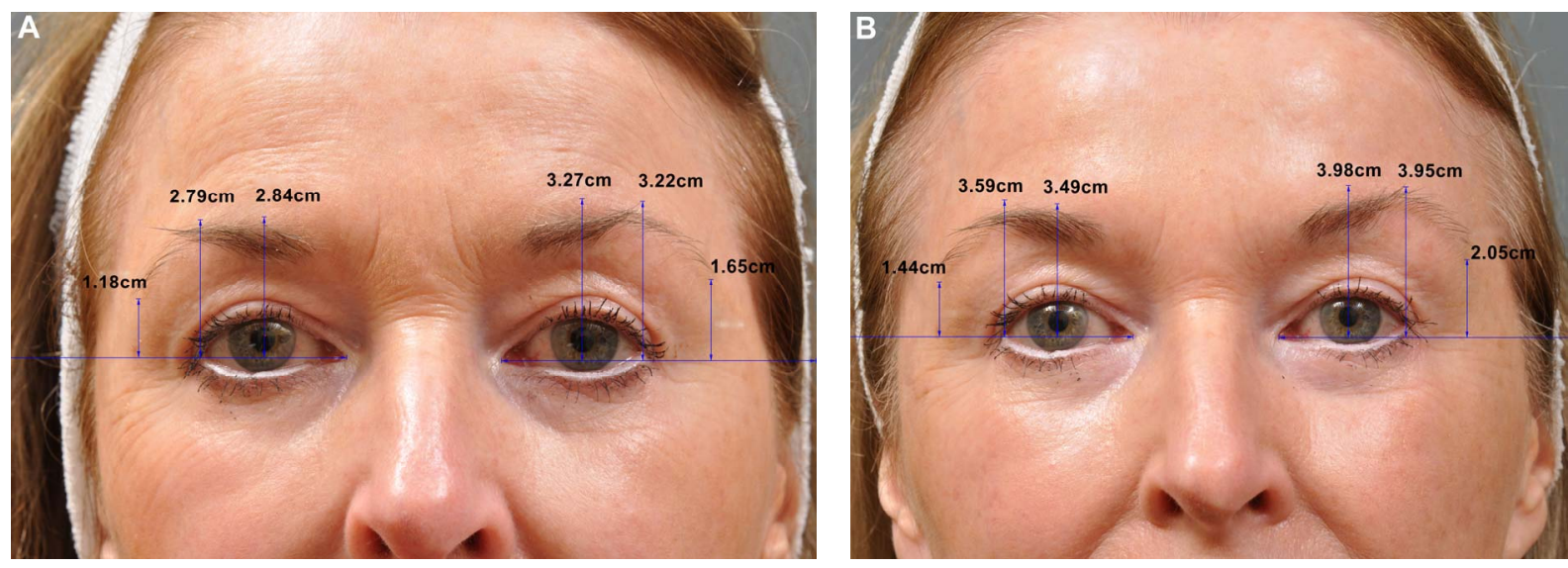

Figure 3. In Panel A, this 67 year old patient presented with crepey periorbital skin and brow ptosis. The lower dermis of the upper lid-brow complex (15 cris-crossing lines), crow's feet area (15 cris-crossing lines) and lower lid (15 cris-crossing lines) was treated with the DS 7 - $30 \mathrm{Nmm}$ transducer (14 mm line; 13 TCPs/line; 0.25 joules/TCP; total 263.2 joules). Thereafter, the upper dermis of the same three areas received treatments (15 lines/site) with the DS $10-1.5 \mathrm{Nmm}$ transducer (14 mm line; 13 TCPs/line; 0.25 joules/TCP; total 146.25 joules). In Panel B, 12 months after treatment, each eyebrow was elevated at its three reference points, measured by the Canfield Matched Orientation Mirror Software. There was a noticeable improvement of the crepey periorbital skin from the baseline image.
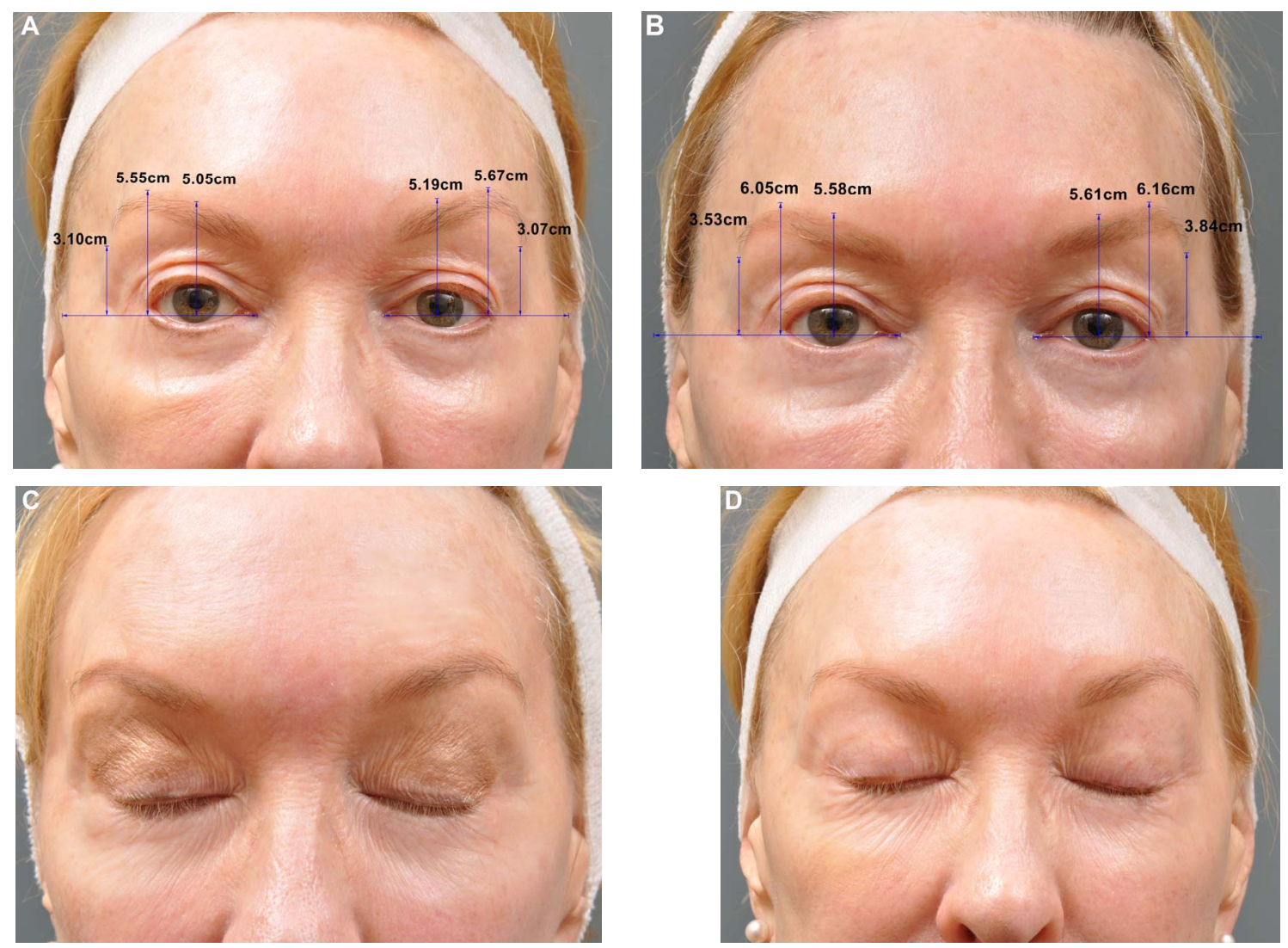

Figure 4. In Panel A, this 60 year old female presented with asymmetric and ptotic right brow relative to her opposite less ptotic side with crepey periorbital skin. Dual dermal treatment was given as listed in the periorbital treatment map with the DS 7 - 3.0 Nmm transducer (14 mm line; 13 TCPs/line; 0.25 joules/TCP; total 263.2 joules) and the DS 10 - 1.5 Nmm transducer (14 mm line; 13 TCPs/line; 0.24 joules/TCP; total 146.25 joules). In Panel B, 12 months after treatment, right brow ptosis was improved from baseline images with elevation of both brows to a more symmetrical level (Mirror Matched Orientation). In Panel C, baseline images showed marked crepey periorbital skin; in Panel D, noticeable improvement of the crepey periorbital skin was documented 12 months after single treatment. 
Table 3. Grading of wrinkling, crepey skin and subdermis to evaluation of clinical 6 month responses.

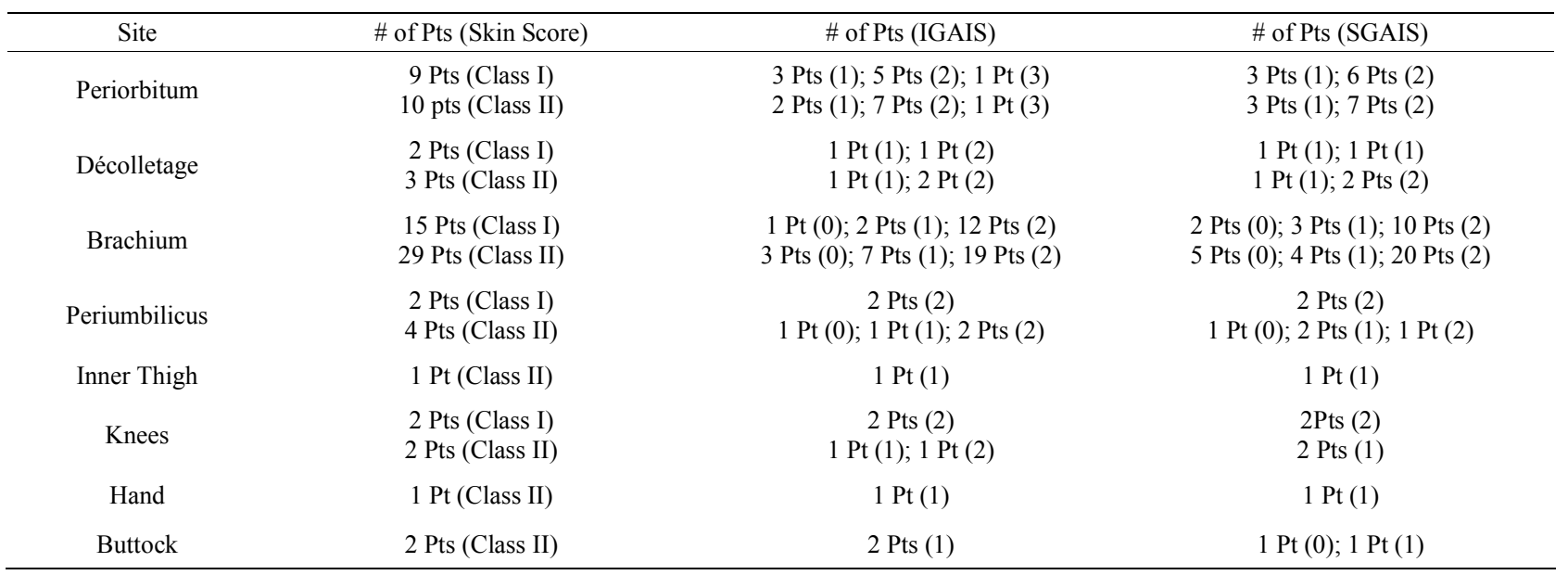
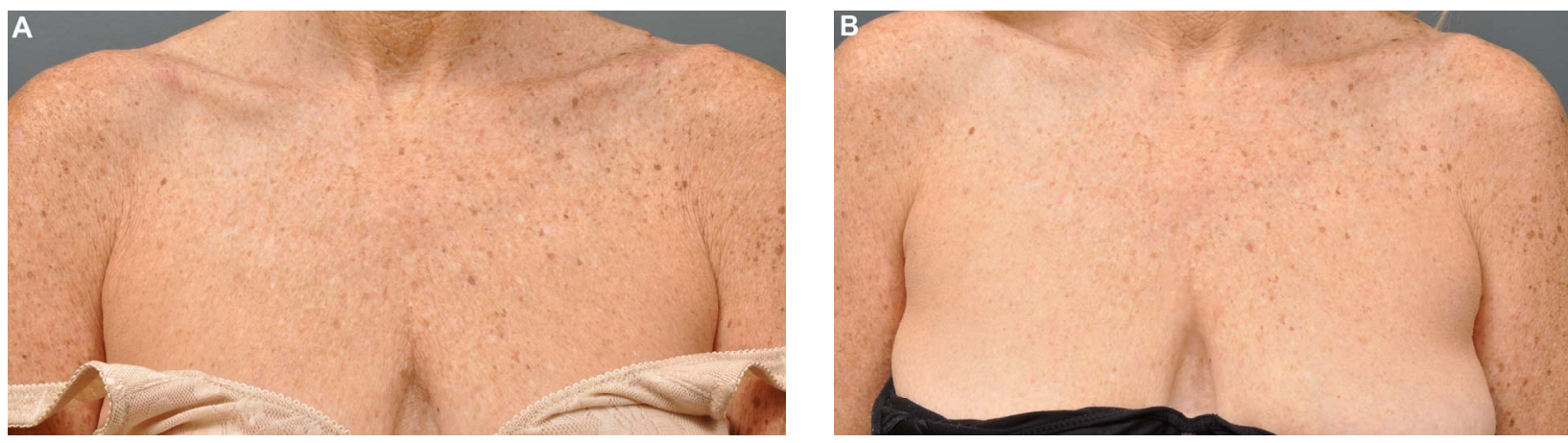

Figure 5. In Panel A, this 50 year old patient had concerns of crepey, pigmented and wrinkled skin in her décolletage and intermammary folds. The exposed region was outlined as an inverted pyramid of twenty-one $2.5 \times 2.5 \mathrm{~cm}$ squares. Each sector received treatments in the lower reticular dermal plane with the DS 7 - $3.0 \mathrm{~mm}$ transducer ( 30 lines; $25 \mathrm{~mm}$ line; $23 \mathrm{TCPs} / \mathrm{line}$; 0.45 joules/TCP; total 6520.5 joules), followed in the upper third of the reticular dermal plane with the DS 10 - 1.5 mm transducer (30 lines; 25 mm line; 23 TCPs/line; 0.25 joules/TCP; total 3622.5 joules). In Panel B, moderate reduction in vertical crinkle lines, superficial pigmentations, thin skin, and also wrinkling within the intermammary folds was observed 9 months after treatment.
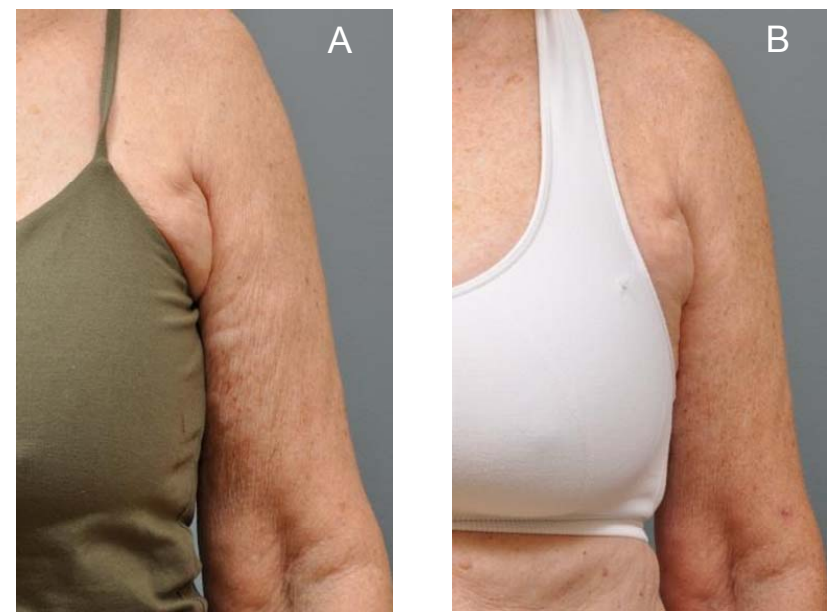

Figure 6. In Panel A, this 65 year old patient presented with generalized creping of the brachial skin. The visible crepey inner brachial skin was marked into sixteen $2.5 \times 2.5 \mathrm{~cm}$ squares. Each sector was treated in the subdermal plane with the DS $4-4.5$ mm transducer (30 lines; $25 \mathrm{~mm}$ line; $17 \mathrm{TCPs} / \mathrm{line} ; 1.2$ joules/TCP, total 9792 joules), followed in the deeper reticular dermal plane with the DS 7 - 3.0 mm transducer (30 lines; 25 mm line; 23 TCPs/line; 0.45 joules/TCP; total 4968 joules). In Panel B, significant improvement was observed 9 months after treatment with smoothing and tightness to the thin crepey skin. 
sites because of uncomfortable pain during treatments that were not ameliorated even by topical anesthetic gels.

Patients assessed their perceived pain levels during the initial part of their treatment prior to requests, if needed, for topical anesthetic gels, selective nerve blocks or local subcutaneous anesthetic infiltrations, as listed in Table 4.

\section{Discussion}

Lax crepey skin and localized subcutaneous fat folds on the body occur not only with chronological aging, but also after photoaging, or sequelae after pregnancy and weight loss. Although surgical procedures address these findings more effectively, they carry inherent risks and require recovery times. Non-invasive thermal devices [7-12] have the potential to denature triple helical chains within collagen fibers between $65^{\circ} \mathrm{C}-70^{\circ} \mathrm{C}$ with immediate recoil and contraction of collagen fibers in the dermis and subcutaneous tissues. Later neocollagenesis and elastogenesis occur at three months with remodeling effects for long-term tissue tightening and improvement of the crepey skin [13-15].

In this preliminary clinical report, non-invasive delivery of microfocused ultrasonic thermal coagulation points to two levels of the dermal skin around the periorbitum,
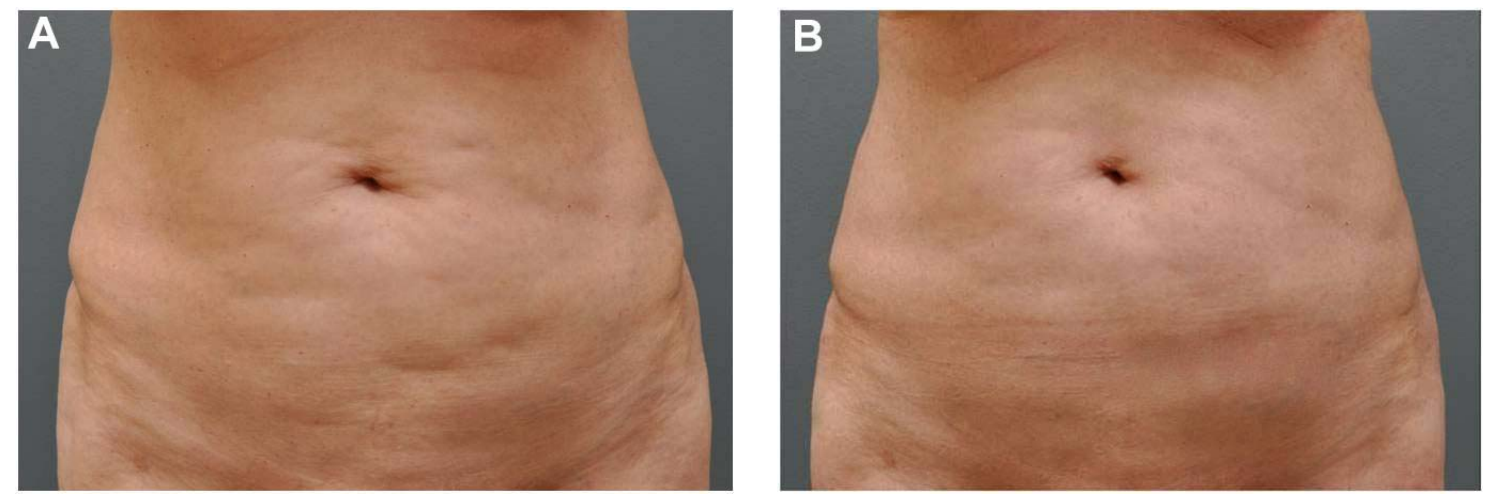

Figure 7. In Panel A, this 72 year old patient (gravida 3, para 3) complained of crepey lines around the periumbilical area and surface irregularities (resembling cellulitic formations) to the remainder of the abdomen. The patient had received no surgical or non-invasive procedures in the past. The periumbilicus and adjacent abdomen was demarcated into twenty-four $2.5 \times 2.5 \mathrm{~cm}$ squares. Each square received treatment to the subdermal plane with the DS $4-4.5 \mathrm{~m}$ transducer (30 lines; $25 \mathrm{~mm}$ line; 17 TCPs/line; 1.2 joules/TCP; total 14,688 joules), and secondly in the deeper reticular dermis with the DS 7 - $3.0 \mathrm{~mm}$ transducer (30 lines; $25 \mathrm{~mm}$ line; 23 TCPs/line; 0.45 joules/TCP; total 7452 joules). The third treatment level consisted of treatment in the upper third of the dermis with the DS 10 - $1.5 \mathrm{~mm}$ transducer (30 lines; 25 mm line; 23 TCPs/line; 0.25 joules; TCP; total 4140 joules). In Panel B, moderate reduction in the crepey wrinkle lines around the umbilicus as well as the surface depressions/folds to the surrounding treated abdomen was observed 1 year after treatment.
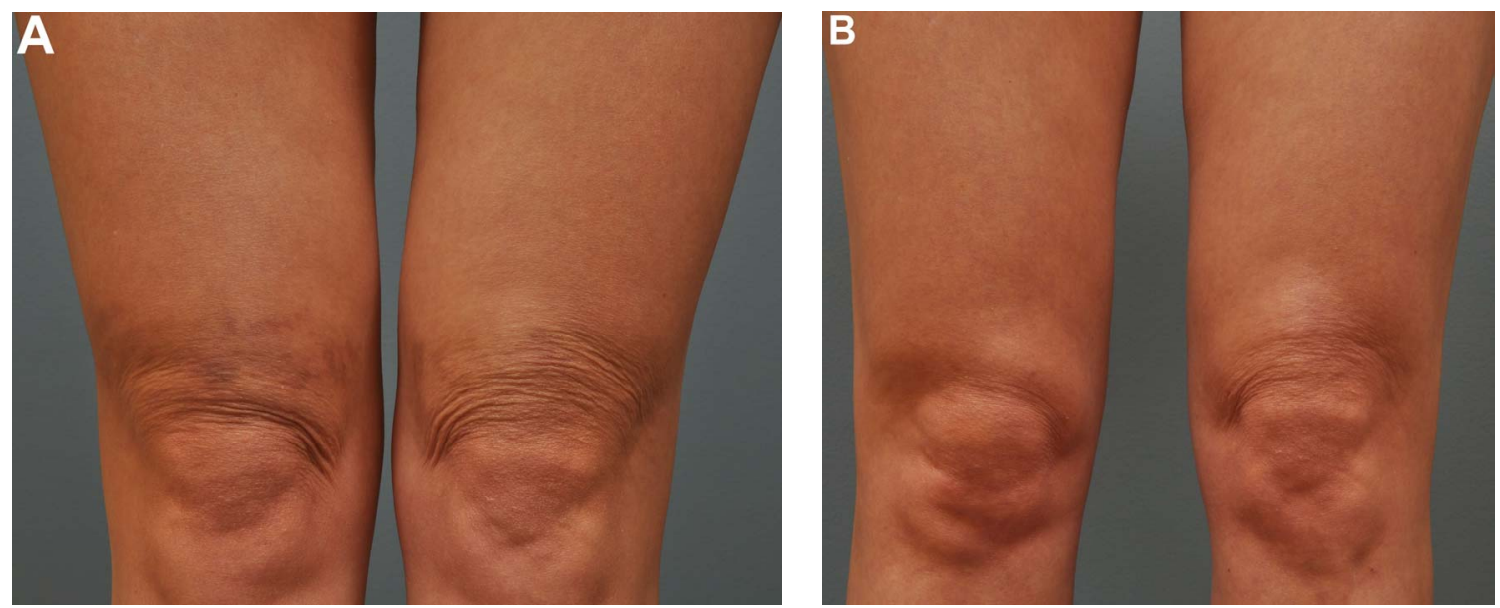

Figure 8. In Panel A, this 39 year old patient sought treatment for crepey transverse lines above the knees. The area of visible lines was marked into twelve $2.5 \times 2.5 \mathrm{~cm}$ squares. Each sector was treated in the subdermal plane with the DS 4 - $4.5 \mathrm{~mm}$ transducer ( 30 lines; 25 mm line; 17 TECPs/line; 1.2 joules/TCP; total 7344 joules) and subsequently treated with the DS $7-3.0 \mathrm{~mm}$ transducer (30 lines; 25 mm line; 23 TCPs/line/ 0.45 joules/TCP; total 3726 joules). In Panel B, marked reduction in the gathered crepey lines was noted 9 months after treatment. 
Table 4. Pain assessment to each treated site: $0=$ no pain $10=$ severe pain.

\begin{tabular}{|c|c|c|c|c|}
\hline Treatment Site & $\begin{array}{c}\text { Number of } \\
\text { Assessed Patients }\end{array}$ & Mild (1 - 4/10) & Moderate $(5-8 / 10)$ & Severe $(9-10 / 10)$ \\
\hline Periorbitum & 19 & 15 & 2 & 2 \\
\hline Décolletage & 5 & 1 & 2 & 2 \\
\hline Brachium & 44 & 10 & 21 & 13 \\
\hline Hand & 1 & 0 & 1 & 0 \\
\hline Periumbilicus & 6 & 0 & 5 & 1 \\
\hline Buttock & 2 & 0 & 1 & 0 \\
\hline Upper Inner Thigh & 1 & 0 & 1 & 0 \\
\hline Knee & 4 & 0 & 3 & 1 \\
\hline
\end{tabular}

appeared to be effective in reduction of wrinkles, crepey and sagging skin for about $1 \frac{1}{2}$ years in all nineteen treated patients. Delayed collagen remodeling is believed to result in a synergistic tightening and elevation of brow-tissues, averaging $1-2 \mathrm{~mm}$ in all 19 treated patients between six weeks to $1 \frac{1}{2}$ years, as determined by the Matched Orientation Function of Mirror Software. The degree of response correlated inversely with the severity of classification on the Fitzpatrick Wrinkle, Fold and Tissue Scale, i.e., the lower the FWFTLS score for skin aging, the greater the response as determined by the Investigator and Subject Global Aesthetic Improvement Scales. A single treatment produced minimal downtime, moderate discomfort and nominal side effects without epidermal injury.

In areas of the décolletages, brachii, periumbilical sites, and knees, dual treatment levels to the dermis and subdermis transformed crepey and wrinkled skin (Class I and II) in the majority of patients to mildmoderate improvements by IGAIS and SGAIS evaluations. Although there were few patients treated in areas of the décolletage, abdomen, and knees, encouraging responses were observed by the third month and continued for a year after a single treatment.

In the few numbers of patients treated in the upper inner thighs, hands and buttocks, results were modest after high energy density treatments were provided to the dermis and subdermis. Significant degrees of thin crepey skin, as often observed in the dorsum of hands and medial thighs, might have contributed to weak responses after a year follow up.

It is important to note that microfocused ultrasound treatments to tissues of the periorbitum and different regions of the body were more sensitive to thermal coagulation points than previously observed in patients who received high density treatments to the face and neck $[1,2]$. Moderate to significant intraoperative pain was experienced most commonly to the décolletage, brachium, knee and periumbilical sites. In order to obtain the greatest potential for clinical responses with higher density en- ergy deposition, the addition of small amounts of local anesthesia in the subcutaneous tissue currently is preferable to reducing the total amount of energy delivered by shortening each treatment line per sector (which, in turn, decreases the number of coagulation points per treatment line) or reducing the energy per thermal coagulation point. Notably, the number and degree of clinical responses to dual levels of treatment appear to be directly related to selection of patients who present with mild-to moderate degrees of skin thinness and laxity and smaller amounts of sagging subcutaneous fat.

The limitations of this preliminary report were the unknown optimal treatment parameters in this first ultrasound experience with the current technology to periorbital tissues and differing body sites. Further innovations in the delivery of increased energy to more structural levels and in improvement in the treatment algorithms (higher densities, more passes, vectored treatment lines, different dermal and subdermal depths) may increase tissue tightening and induction of more collagenesis to renew thin and crepey dermis without increasing significant adverse events, costs, and delivery times. Needless to say, greater patient enrollment and longer follow up evaluations by volumetric and measurement advances are needed. In the near future, this novel energy device may be able to visualize and treat more specific structural levels than currently available for tissue tightening but also rejuvenation, volumetric reduction and treatment of troublesome dermal adnexal structures.

\section{Conclusions}

Microfocused ultrasound for nonablative thermal tightening and rejuvenation of thin crepey tissue to the periorbitum and body sites appears to be safe and moderately effective by outcome evaluations in this small cohort of patients limited to mild-to moderate degrees of tissue wrinkling and laxity. Responders experienced positive effects up to $1 \frac{1}{2}$ years, especially to crepey skin in the periorbitum, décolletage, brachium and knees. All patients commonly experienced transitory mild swelling and erythema. 
Most patients reported moderate-severe pain during treatments in differing body sites that required either local nerve blocks or injections within the subcutaneous tissue. The cumulative effects of upper and lower dermal treatments for improvements in crepey wrinkled skin are encouraging but will require further clinical and objective validations.

\section{Acknowledgements}

The authors wish to thank Margaret Gaston, BS, for statistical and photographic assistance.

\section{Disclosures}

Dr. Sasaki is a consultant to Ulthera, Inc. and received limited funding under an unrestricted research grant for the studies. Registered Nurse Ana Tevez has no financial disclosures.

\section{REFERENCES}

[1] M. Alam, L. E. White, N. Martin, et al., "Ultrasound Tightening of Facial and Neck Skin: A Rater-Blinded Prospective Cohort Study," Journal of the American Academy of Dermatology, Vol. 62, No. 2, 2009, pp. 262-269. doi:10.1016/j.jaad.2009.06.039

[2] G. H. Sasaki and Tevez, "Focused Imaged Ultrasound: Clinical Efficacy and Safety (2 Year Experience)," Aesthetic Surgery Journal, Vol. 32, No. 5, 2012.

[3] W. M. White, I. R. S. Makin, P. G. Barthe, et al., "Selective Creation of Thermal Injury Zones in the Superficial Musculoaponeurotic System Using Intense Ultrasound Therapy," Archives of Facial Plastic Surgery, Vol. 9, No. 1, 2007, pp. 922-929.

[4] W. M. White, I. R. S. Makin, M. H. Slayton, et al., "Selective Transcutaneous Delivery of Energy to Porcine Soft Tissues Using Intense Ultrasound (IUS)," Lasers in Surgery and Medicine, Vol. 40, No. 2, 2008, pp. 67-75. doi:10.1002/lsm.20613

[5] R. E. Gliklich, W. M. White, M. H. Slayton, et al., "Clinical Pilot Study of Intense Ultrasound Therapy to Deep Dermal Facial Skin and Subcutaneous Tissues," Archives of Facial Plastic Surgery, Vol. 9, No. 2, 2007, pp. 88-95. doi:10.1001/archfaci.9.2.88

[6] D. Manstein, G. S. Herron and R. K. Sink, "Fractional Photothermolysis: A New Concept for Cutaneous Re- modeling Using Microscopic Patterns of Thermal Injury," Lasers in Surgery and Medicine, Vol. 34, 2004, pp. 426438. doi:10.1002/1sm.20048

[7] G. H. Sasaki, H. M. Travis and B. Tucker, "Fractional $\mathrm{CO}_{2}$ Laser Resurfacing of Photoaged Facial and Non-Facial Skin: Histologic and Clinical Results and Side Effects," Journal of Cosmetic and Laser Therapy, Vol. 11, No. 4, 2009, pp. 190-201. doi: $10.3109 / 14764170903356465$

[8] T. S. Alster and J. R. Lupton, "Nonablative Cutaneous Remodeling Using Radiofrequency Devices," Clinics in Dermatology, Vol. 25, 2007, pp. 487-491. doi:10.1016/j.clindermatol.2007.05.005

[9] C. C. Dierickx, "The Role of Deep Heating for Noninvasive Skin Rejuvenation," Lasers in Surgery and Medicine, Vol. 38, No. 9, 2006, pp. 799-807. doi: $10.1002 / 1 \mathrm{sm} .20446$

[10] N. Sadick, "Tissue Tightening Technologies: Fact or Fiction," Aesthetic Surgery Journal, Vol. 28, 2008, pp. 180-188.

[11] M. L. Elsaie, "Cutaneous Remodeling and Photorejuvenation Using Radiofrequency Devices," Indian Journal of Dermatology, Vol. 54, No. 3, 2009, pp. 201-205. doi:10.4103/0019-5154.55625

[12] D. J. Maitland and J. T. Walsh, "Quantitative Measurements of Linear Birefringence during the Heating of Native Collagen," Lasers in Surgery and Medicine, Vol. 20, No. 3, 1997, pp. 310-318. doi:10.1002/(SICI)1096-9101(1997)20:3<310::AID-LSM 10>3.0.CO;2-H

[13] S. P. Arnoczky and A. Aksan, "Thermal Modification of Connective Tissues: Basic Science Considerations and Clinical Applications," Journal of the American Academy of Orthopaedic Surgeons, Vol. 8, No. 5, 2000, pp. 305313.

[14] B. M. Hantash, A. A. Ubeid, H. Chang, et al., "Bipolar Fractional Radiofrequency Treatment Induces Neoelastogenesis and Neocollagenesis," Lasers in Surgery and Medicine, Vol. 41, No. 1, 2009, pp. 1-9. doi:10.1002/1sm.20731

[15] D. Shoshani, E. Markovitz, S. J. Monstrey, et al., "The Modified Fitzpatrick Wrinkle Scale: A Clinical Validated Measurement Tool for Nasolabial Wrinkle Severity Assessment," Dermatologic Surgery, Vol. 34, No. S1, 2008, pp. S85-S91. doi:10.1111/j.1524-4725.2008.34248.x 